\title{
Effect of acute isooxic hypercapnia on oxidative activity of systemic neutrophils in endotoxemic rabbits
}

\author{
HANNA BILLERT ${ }^{1}$ EWA BEDNAREK ${ }^{1}$, KRZYSZTOF KUSZA², MIROSEAW PONICHTER ${ }^{l}$, \\ MACIEJ KURPISZ ${ }^{3}$
}

${ }^{1}$ Department of Experimental Anaesthesiology, Chair of Anaesthesiology and Intensive Therapy, Poznan University of Medical Sciences, Poznan, Poland

${ }^{2}$ Clinics of Anaesthesiology and Intensive Therapy, Chair of Anaesthesiology and Intensive Therapy, Poznan University of Medical Sciences, Poznan, Poland

${ }^{3}$ Institute of Human Genetics, Polish Academy of Sciences, Poznan, Poland

\begin{abstract}
Introduction: Whether carbon dioxide $\left(\mathrm{CO}_{2}\right)$ affects systemic oxidative phenomena under conditions of endotoxemia is not sufficiently clarified. The study aimed to assess the impact of moderate acute hypercapnia on the respiratory burst of circulating neutrophils in mechanically ventilated endotoxemic rabbits.

Material and methods: Twenty-four endotoxemic rabbits were mechanically ventilated with standard or $\mathrm{CO}_{2}$-enriched gas mixture in order to obtain isooxic hypercapnia. At a baseline point and following 180 min of hypercapnic ventilation, luminol-dependent chemiluminescence $(C L)$ of circulating neutrophils and serum 2-thiobarbituric acid reactive substance (TBARS) concentrations were measured. Throughout the study, leukocyte and neutrophil counts, $p H$ status, circulatory parameters and body temperature were also assessed.

Results: Following 180 min of hypercapnic ventilation, opsonized zymosan (OZ)-stimulated neutrophils showed lower CL vs. the control group $(p=0.004)$. Other parameters studied were not affected.

Conclusions: Short-term isooxic hypercapnia in endotoxemic rabbits preserves circulating neutrophil count pattern and reactive oxygen species (ROS) generation, but it may reduce phagocytosis.
\end{abstract}

Key words: endotoxemia, hypercapnia, respiratory burst, circulating neutrophils.

(Cent Eur J Immunol 2021; 46 (1): 47-53)

\section{Introduction}

Hypercapnia may influence the clinical course in septic settings, but its definite impact is still under ongoing discussion [1, 2]. Independently of ventilation parameters and in a $\mathrm{pH}$-dependent or independent manner, carbon dioxide $\left(\mathrm{CO}_{2}\right)$ may exert either tissue protective or deleterious effects [3], corresponding to its anti- or proinflammatory properties, and improving [4] or not [5] clinical outcomes. Specifically, in septic conditions hypercapnia was reported to increase survival [6], even in immunocompromised patients [7]. However, in patients with moderate or severe ARDS worse outcomes were observed [8]. These partly conflicting results seem to be due to cohort differences involving severity of basal clinical condition, stage of disease, type of pathogen, and organ failure. For the definite impact of hypercapnia, its cardiocirculatory effects and complex immune implications may be crucial. Whether, under septic conditions and coexisting hypercapnia, neutrophil performance could contribute to the outcome remains controversial [9-11].
Neutrophils, key players in inflammation, recognize pathogen or other 'danger' signals and initiate a number of downstream signaling pathways and effector function to combat infection. Generation of reactive oxygen species (ROS), mainly by the respiratory burst activity of NADPH oxidase (NOX2), is critically important not only for pathogen elimination but also for regulation of the inflammatory response and restoring homeostasis under septic challenge [9]. $\mathrm{CO}_{2}$ directly inhibits neutrophil NOX2 activity, ROS generation, phagocytosis and microbial killing $[10,12]$, yet it may occasionally induce oxidative stress [13]. In vivo, neutrophil adaptation mechanisms to changing environmental conditions are less explored. While modulating effects of hypercapnia were demonstrated for neutrophils that had migrated to effector organs, ROS generation by the systemic neutrophil population under isooxic hypercapnia was preserved [14]. However, under concomitant hypoxia, suppression of neutrophil oxidative capacity occurred [15]. Whether in septic conditions elevated partial pressures of $\mathrm{CO}_{2}$ would influence oxidative

Correspondence: Prof. Maciej Kurpisz, Institute of Human Genetics, Polish Academy of Sciences, 32 Strzeszynska St., 60-479 Poznan,

Poland, e-mail: kurpimac@man.poznan.pl

Submitted: 18.10.2019; Accepted: 27.08.2020 
activity of systemic neutrophils, strongly associated with the overall systemic inflammatory reaction [16], is not sufficiently clarified.

Therefore, the study aimed to assess in vivo the respiratory burst capacity of circulating neutrophils in endotoxemic rabbits subjected to isooxic hypercapnia and its influence on the peripheral blood cell pattern.

\section{Material and methods}

Having the approval of the Local Ethic Committee for Animal Investigations, the experiments were carried out on 24 male Chinchilla rabbits weighing $3.8 \pm 0.4 \mathrm{~kg}$. Animals were housed under standard conditions with free access to chow and tap water; they were fasted on the day of experiment. Rabbits' care followed the recommendations of the Helsinki Declaration and the regulations of Polish Animal Protection Law. The rabbit is a suitable experimental model for both mechanical ventilation and endotoxemia studies. Among non-primate species, rabbit shows the highest amino acid similarity to humans regarding lipopolysaccharide (LPS) sensing Toll-like receptor 4 (TLR4) [17].

\section{Experimental protocol}

A 19G intravenous cannula was inserted into the right ear marginal vein. Anaesthesia was induced intravenously by pentobarbital, $40 \mathrm{mg} / \mathrm{kg}$, and maintained with subsequent continuous infusion, $25 \mathrm{mg} / \mathrm{kg} / \mathrm{h}$. Rabbits were intubated with a cuffed endotracheal tube of $3.5 \mathrm{~mm}$ internal diameter. Muscle relaxation was obtained by intravenous pancuronium $1 \mathrm{mg}$, with subsequent hourly supplementation. Rabbits were mechanically ventilated by a volume-cycled animal ventilator (Zimmermann, WGL, Germany) with tidal volume (TV) of $10 \mathrm{ml} / \mathrm{kg}$ and ventilation frequency adjusted to achieve a $\mathrm{PaCO}_{2}$ of $35-45 \mathrm{mmHg}$ (4.7-6 kPa), $\mathrm{F}_{\mathrm{i}} \mathrm{O}_{2}-0.40$.

While intubated, animals were i.v. administered with lipopolysaccharide of Escherichia coli 0111:B4 (Sigma) $20 \mathrm{mg} / \mathrm{kg}$ over $10 \mathrm{~min}$. At this dose, there is observed leukocyte sequestration in lung capillaries and a systemic blood pressure decrease, but no lung injury occurs (that would require doses exceeding $500 \mathrm{mg} / \mathrm{kg}$ ) [18].

After 30 min of stabilization, rabbits were randomized to two groups: normocapnia $(n=12)$ and hypercapnia $(n=12)$. $\mathrm{PaCO}_{2}$ was $35-45$ and $60-68 \mathrm{mmHg}$, respectively. A moderate, clinically acceptable degree of the $\mathrm{PaCO}_{2}$ increase (not exceeding $68 \mathrm{mmHg}$ ) [19] was applied as higher partial pressure might potentially implicate augmentation of tissue injury via nitrosative mechanisms and undesirably interfere with local organ blood flow [20]. The carbon dioxide concentration in the range of 66-75 $\mathrm{mmHg}$ has been recently demonstrated to be most strongly associated with improved survival in emergency department mechanically ventilated patients (aOR 3.18) [4]. Hypercapnia was obtained by adding $\mathrm{CO}_{2}$ to the respiratory mixture.
Arterial access $(17 \mathrm{G})$ to the right ear median artery was established allowing arterial pressure continuous recording and drawing blood samples for gas and acid base analysis, and cellular studies. Continuous monitoring involved mean arterial pressure (MAP), ECG, rectal temperature, end-tidal $\mathrm{CO}_{2}$ and inspiratory $\mathrm{O}_{2}$ fraction (Anaesthetic Gas Monitor Type 1304, Brueel \& Kijaer, Denmark). Arterial blood $\mathrm{PaCO}_{2}, \mathrm{PaO}_{2}$ and $\mathrm{pH}$ were determined at a baseline point (BL) and at the $30^{\text {th }}, 60^{\text {th }}, 120^{\text {th }}$ and $180^{\text {th }}$ minute of hypercapnic or normocapnic CMV (Ciba Corning Analyser 248, Ciba Corning, England).

\section{Neutrophil studies}

Neutrophils were separated from the whole peripheral blood drawn at a baseline point and following $180 \mathrm{~min}$ of hypercapnia and processed using hydroxyethyl cellulose (HEC) and Percoll according to Issekutz [21]. Briefly, the whole blood samples with $2 \%$ EDTA were mixed with $1 \%$ HEC (Fluka Chemicals) and red blood cells were allowed to sediment for $60 \mathrm{~min}$. Leukocyte-rich plasma was then harvested, layered over $68 \%$ Percoll (Sigma) and spun down at $400 \mathrm{~g}, 4^{\circ} \mathrm{C}$ for $20 \mathrm{~min}$. Then, neutrophils were washed and the remaining erythrocytes were lysed with $0.84 \% \mathrm{NH}_{4} \mathrm{Cl}$. Then, the cells were repeatedly washed and resuspended in Tyrode's solution, $\mathrm{pH}$ 7.2; cell preparations contained about $97 \%$ of viable neutrophils (Trypan blue exclusion assay).

Luminol-dependent chemiluminescence (CL) according to Allen et al. reflecting both intra- and extracellular ROS generation was measured by a 1250 LKB luminometer (Bioorbit, Denmark) with minor modifications [22]. The reaction mixture contained $5 \times 10^{5}$ cells, $0.15 \mathrm{mM}$ luminol (Sigma), and either serum (FCS, Sigma; $1 \mathrm{mg} / \mathrm{ml}$ ), opsonized zymosan (OZ, Sigma), $10^{-5} \mathrm{M}$ N-formyl-methionyl-leucyl-phenylalanine (fMLP, Sigma) or $100 \mathrm{ng} / \mathrm{ml}$ phorbol 12-myristate 13-acetate (PMA, Sigma). CL was recorded at $37^{\circ} \mathrm{C}$ for $60 \mathrm{~min}$ and data integrated. Respiratory burst on stimulation with $\mathrm{OZ}$ reflects phagocytosis. $\mathrm{OZ}$ was prepared according to Labedzka et al. [23].

\section{Oxidative stress}

Reflecting oxidative stress, 2-thiobarbituric acid reactive substances (TBARS), which consist of lipid hydroperoxides and aldehydes and parallel concentrations of malondialdehyde (MDA), were estimated by the spectrophotometric method of Macfarlane [24]. Serum (100 ml) was mixed with $1 \mathrm{M}$ suprachloric acid and $42 \mathrm{mM}$ thiobarbituric acid and heated at $100^{\circ} \mathrm{C}$ for $30 \mathrm{~min}$. Then, after obtaining room temperature the samples were spun down and the absorbance was estimated by $\lambda=507,532$, and $557 \mathrm{~nm}$ (Specord M-40, Germany).

End result was estimated as: $\mathrm{cA}_{532} / 0.152$ (molar absorbance index for MDA $) \times$ volume $(0.1 \mathrm{ml})$; where $\mathrm{cA}_{532}$ $($ corrected absorbance measured $)=A_{532}-1 / 2\left(A_{507}-A_{557}\right)$. 


\section{Statistics}

Statistical analysis was performed using GraphPad Prism Version 6 (La Jolla, USA). Data were expressed as the mean \pm standard error of the mean. Statistical comparisons between the groups were performed using the Mann-Whitney $U$-test. Differences between time points were assessed using the Wilcoxon signed rank test and Friedman's ANOVA with post hoc Dunn's multiple comparisons. Correlations were performed by means of the Spearman rank test. Statistical significance was set at $p<0.05$.

\section{Results}

\section{Neutrophil studies}

At the $30^{\text {th }}$ min of hypercapnia both leukocyte and neutrophil counts were strikingly lower than at baseline (Fig. 1A, B) and remained decreased until the end of the experiment. Hypercapnic and normocapnic rabbits followed a similar cell count pattern, $p>0.05$.
Acute hypercapnia did not result in significant differences in neutrophil CL values as compared to normocapnic conditions (Fig. 1A-D). Following $180 \mathrm{~min}$ of hypercapnic ventilation, neutrophil CL also remained stable in unstimulated and PMA- and fLMP-stimulated neutrophils (Fig. 1A, C, D), whereas under OZ stimulation a significant decrease could be noted ( $p=0.004$; Fig. 1B).

\section{Oxidative stress evaluation}

TBARS concentrations were comparable between the groups and timepoints ( $p>0.05$, Fig. 1G).

\section{Blood gas and acid-base status, hemodynamic measurements and body temperature evaluation}

Mean results of the arterial blood $\mathrm{pH}, \mathrm{PaCO}_{2}$, and $\mathrm{PaO}_{2}$ values at the time points selected are presented in Figure 2A-C. Hypercapnic rabbits demonstrated acidosis beginning at the $30^{\text {th }} \mathrm{min}$, which could also be observed in normocapnic rabbits from the $120^{\text {th }} \mathrm{min}$ (Fig. 2A). Conditions of isooxia were assured (Fig. 2C).
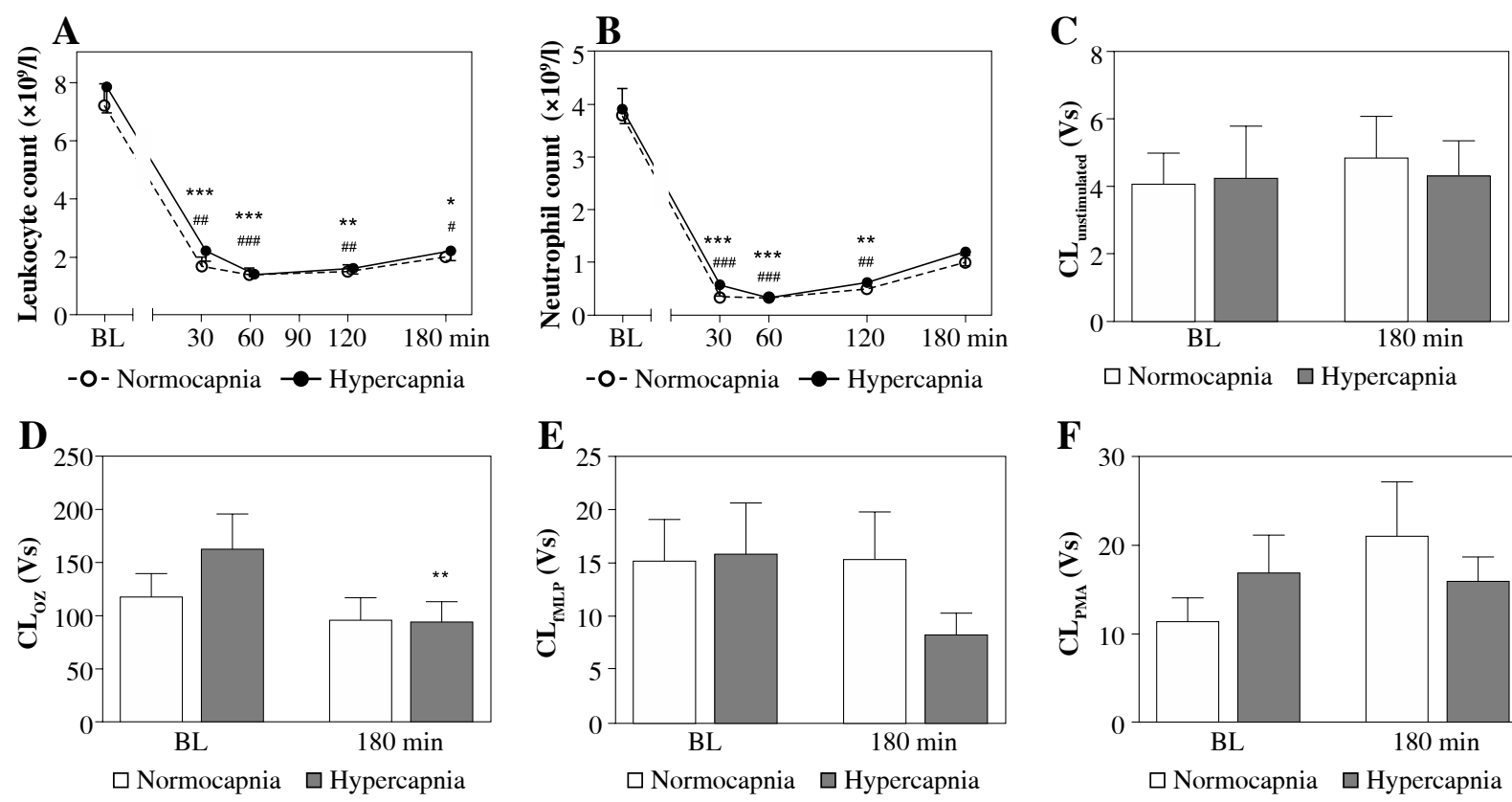

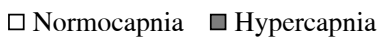

F

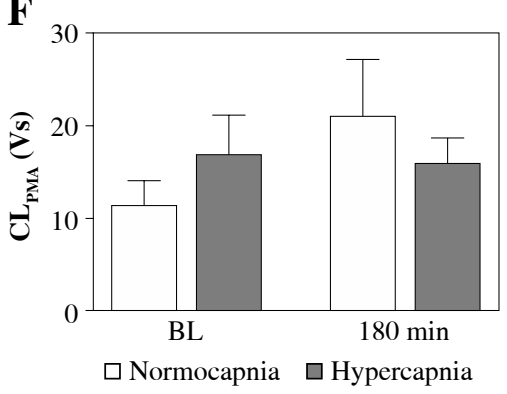

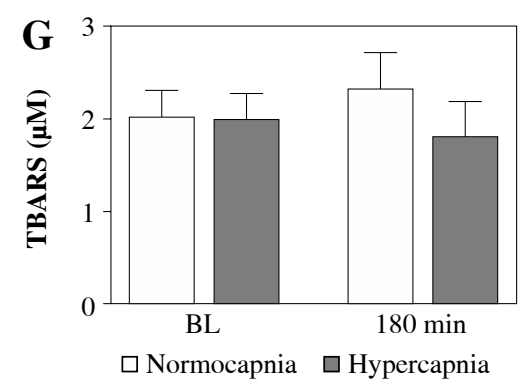

Fig. 1. Peripheral blood leukocyte (A) and neutrophil counts (B) over experimental period, luminol-dependent chemiluminescence (CL) of isolated peripheral blood neutrophils unstimulated $(\mathbf{C})$, stimulated with (D) opsonized zymosan (OZ, $1 \mathrm{mg} / \mathrm{ml}),(\mathbf{E}) \mathrm{N}$-formyl-methionyl-leucyl-phenylalanine (fMLP, $106 \mathrm{M}$ ), (F) phorbol 12-myristate 13-acetate (PMA, $100 \mathrm{ng} / \mathrm{ml}$ ), and 2-thiobarbituric acid reactive substance (TBARS) concentrations (G) in endotoxemic rabbits at a baseline (BL) and after 180 min of mechanical ventilation under conditions of isooxic normocapnia (white points, dotted line, white bars) or hypercapnia (black points, solid line, gray bars). $\mathrm{M} \pm \mathrm{SEM}$; ${ }^{* * *} p<0.001, * * p<0.01,{ }^{*} p<0.05$ vs. BL in normocapnia group; ${ }^{\# \#} p<0.001,{ }^{\#} p<0.01,{ }^{\#} p<0.05$ vs. BL in normocapnia group. D) $* *$ denotes exact $p$ value $=0.004$ (Mann-Whitney $U$-test $)$ 
A

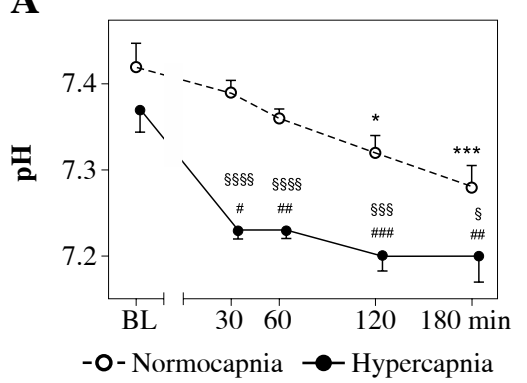

D

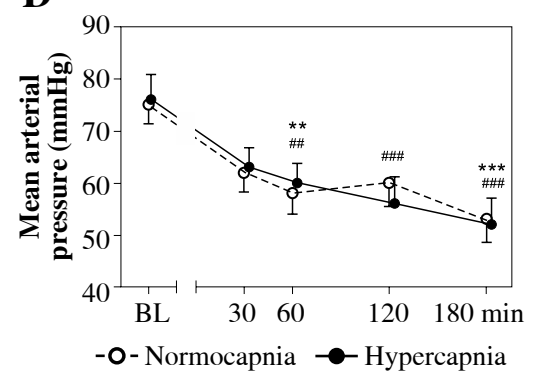

B

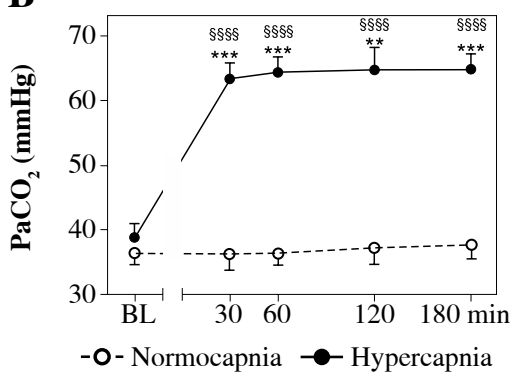

$\mathbf{E}$

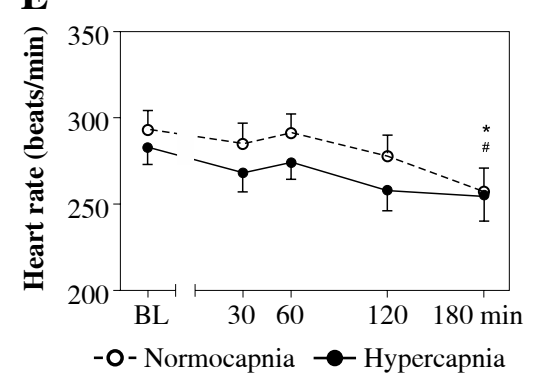

C
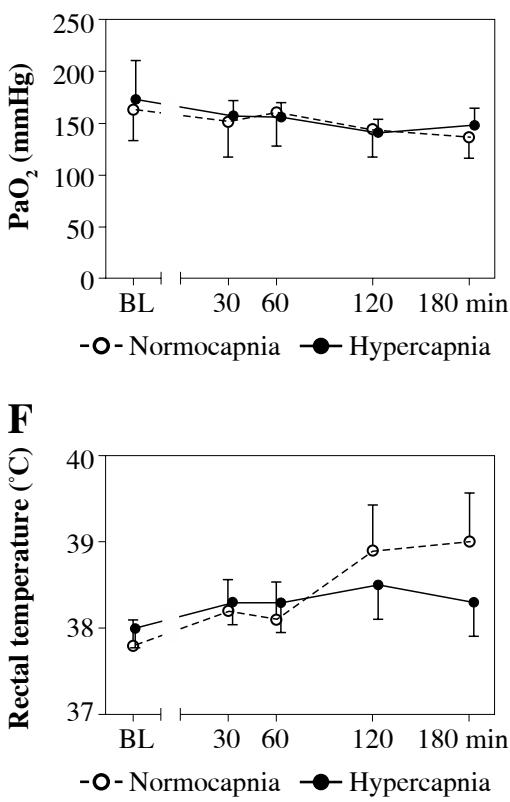

Fig. 2. Arterial blood pH (A), $\mathrm{PaCO}_{2}(\mathbf{B}), \mathrm{PaO}_{2}(\mathbf{C})$, mean arterial pressure (D), heart rate (E), and rectal temperature (F) in endotoxemic rabbits mechanically ventilated under normo- and hypercapnic conditions at a baseline (BL) and following $30,60,90$, and $180 \mathrm{~min} . \mathrm{M} \pm \mathrm{SEM} ;{ }^{* * *} p<0.001,{ }^{* *} p<0.01,{ }^{*} p<0.05 \mathrm{vs}$. BL in normocapnia group; ${ }^{\# \#} p<0.001, \# p<0.01,{ }^{\#} p<0.05$ vs. BL in normocapnia group. A, B) Significant differences between the groups, exact $p$ values are as follows: ${ }^{\circledR} \$ \$ 8 p<0.0001,{ }^{8 \$ 8} p=0.0005,{ }^{8} p=0.0238$ vs. normocapnia group (Mann-Whitney $U$-test)

Mean arterial pressure (MAP) and heart rate were comparable between the groups throughout the study period (Fig. 2D,E). In the normocapnia group arterial pressure and heart rate were lower at the end of the experiment. In hypercapnic rabbits a pressure decrease could be detected as soon as at the $60^{\text {th }}$ min of hypercapnia with no significant changes in heart rate throughout the experiment.

Rectal temperature was comparable for the groups and the timepoints measured (Fig. 2F), $p>0.05$.

\section{Associations between neutrophil CL, neutrophil count, $\mathrm{pH}$ and blood gas values in endotoxemic rabbits}

At the end of the study period independently of partial $\mathrm{CO}_{2}$ pressures, $\mathrm{CL}$ of unstimulated and stimulated circulating neutrophils appeared to inversely correlate with the cell count (Table 1). In both studied groups the association was most pronounced regarding unstimulated neutrophils and could also be confirmed in OZ-stimulated cells. A significant correlation between the neutrophil count and CL of the PMA-stimulated cells was observed under normocapnic conditions; in hypercapnic rabbits the association was significant under fMLP stimulation.

Increased partial pressures of $\mathrm{CO}_{2}$ were negatively associated with CL of the fMLP-stimulated neutrophils after 180 min of hypercapnia; at that time the decreased $\mathrm{pH}$ in hypercapnic animals nearly positively correlated with both fMLP- and PMA-stimulated neutrophils.

\section{Discussion}

The current study demonstrated that short-term isooxic hypercapnia of moderate degree in the mechanically ventilated, endotoxemic rabbits neither modulates peripheral leukocyte and neutrophil count patterns nor changes extra- and intracellular ROS generation by circulating neutrophils. However, in hypercapnic animals it could induce phagocytosis impairment.

\section{Neutrophil count}

Depending on experimental conditions, systemic endotoxin administration may increase or decrease the neutrophil count. In this study, quite in line with previous findings in endotoxemic rabbits, a rapid neutropenia persisting until the end of the study was observed [18]. In rabbits, expression of the critical for LPS sensing TLR4 is the highest in lungs and bone marrow [17]. Endotoxemic neutropenia results from preferable lung sequestration of the bone marrow-released, less deformable neutrophils of mixed maturity and this is due to L-selectin activation [18, 25].

In the present study, a similar degree of neutropenia between normo- and hypercapnic animals was observed. 
Table 1. Spearman rank correlations between neutrophil chemiluminescence, cell count, $\mathrm{pH}$ and blood gas values ( $\mathrm{pH}$, $\mathrm{PaCO}_{2}, \mathrm{PaO}_{2}$ ) in endotoxemic rabbits following $180 \mathrm{~min}$ of hypercapnic mechanical ventilation and respective control animals

\begin{tabular}{|c|c|c|c|c|}
\hline \multicolumn{5}{|c|}{ Neutrophil chemiluminescence } \\
\hline & Unstimulated & OZ-stimulated & fMLP-stimulated & PMA-stimulated \\
\hline \multicolumn{5}{|c|}{ Normocapnia } \\
\hline Neutrophil count & $-0.829(0.003)$ & $-0.667(0.032)$ & -0.564 & $-0.782(0.038)$ \\
\hline $\mathrm{pH}$ & 0.091 & 0.212 & 0.286 & 0.143 \\
\hline $\mathrm{PaCO}_{2}$ & -0.109 & 0.274 & 0.393 & 0.143 \\
\hline $\mathrm{PaO}_{2}$ & $-0.745(0.013)$ & -0.479 & -0.179 & -0.464 \\
\hline \multicolumn{5}{|c|}{ Hypercapnia } \\
\hline Neutrophil count & $-0.774(0.009)$ & $-0.646(0.043)$ & $-0.898(0.002)$ & -0.159 \\
\hline $\mathrm{pH}$ & 0.353 & 0.151 & $0.703(0.078)$ & $0.634(0.066)$ \\
\hline $\mathrm{PaCO}_{2}$ & -0.343 & -0.100 & $-0.929(0.003)$ & -0.343 \\
\hline $\mathrm{PaO}_{2}$ & -0.100 & -0.300 & -0.071 & -0.100 \\
\hline
\end{tabular}

Depending on experimental model, hypercapnia was reported to increase or not neutrophil lung sequestration in septic conditions $[1,10]$. In our study, concomitant acidosis decreasing L-selectin expression could have counteracted possibly increased neutrophil pulmonary sequestration in hypercapnic endotoxemic rabbits $[18,26]$.

\section{ROS generation}

In line with our previous observations in rabbits not treated with endotoxin, we found that neutrophil ROS generation remained stable under short-term moderate increase of systemic partial pressures of $\mathrm{CO}_{2}$ and an inflammatory microenvironment [14]. Nor did endotoxin administration at the dose applied affect systemic neutrophil oxidative capacity, following an $180 \mathrm{~min}$ observation period. Depending on experimental conditions, endotoxin can either suppress or stimulate oxidative activity of systemic neutrophils [27, 28]. In this study, after $210 \mathrm{~min}$ (including stabilization period) a moderate, single dose of LPS ( $\mathrm{T} 1 / 2=53 \mathrm{~min}$ ) could be insufficient to cause any priming effect of LPS itself or secondary priming factors, e.g. tumor necrosis factor $\alpha(\mathrm{TNF}-\alpha)[18,29]$.

Decreased CL values of OZ stimulated circulating neutrophils following $180 \mathrm{~min}$ of hypercapnia could indicate phagocytosis impairment; however, no differences between the groups at this timepoint were found. Of note, a decreasing tendency in the fMLP-stimulated CL reflecting antibacterial activity following $180 \mathrm{~min}$ of hypercapnia turned out to be insignificant. However, G-protein-coupled receptor (GPCR) signaling mediating fMLP-induced oxidative burst is complex and involves a number of downstream proteins and negative feedback loops that may implicate a substantial dispersion of the final results [30]. Additionally, coexisting hypercapnia may influence turn- over of phosphatidic acid critical for the fMLP-stimulated CL neutrophil response [31]. Neutrophil phagocytosis suppression that may also result in deficient antimicrobial defense at the early stage of infection was observed under exposure to $\mathrm{CO}_{2}$, cold stress and hypoxia [15], and following peritoneal $\mathrm{CO}_{2}$ insufflation [32]. On the other hand, probability of developing ARDS in trauma patients was associated with greater ROS production by the OZ-stimulated neutrophils [33]. Therefore, the phenomenon observed in this study could be desirable in terms of the possible protective effect of moderate hypercapnia regarding lung injury under endotoxemia. Interestingly, in ARDS patients severe hypercapnia predicted worse clinical outcome [8].

In this study, under isooxic conditions a moderate increase of $\mathrm{CO}_{2}$ partial pressures did not impact either fMLP- or PMA-stimulated respiratory burst. In an oncologic cohort, PMA-stimulated neutrophil respiratory burst was suggested to be a reliable predictor of susceptibility to infection [34]. In vitro, following $15 \mathrm{~min}$ and $2 \mathrm{~h} \mathrm{ex}$ posure to $100 \% \mathrm{CO}_{2}$, decreased ROS generation by the PMA-stimulated neutrophils was reported [12].

We also found that neutrophil count negatively correlated with ROS generation. The neutropenic population represents unsequestered cells with possibly lower L-selectin expression; an inverse relationship between neutrophil L-selectin shedding and respiratory burst was previously reported [35].

Earlier research attributed the effect of hypercapnia attenuating neutrophil ROS generation to coexisting acidosis [3]. In this study, despite differences in systemic $\mathrm{pH}$ decrease, deeper in hypercapnic rabbits, mean $\mathrm{pH}$ values in both groups were still in the clinically acceptable range (> 7.15) [19]. A value of 7.2 was previously reported to be optimal regarding the respiratory burst, but still phago- 
cytosis of opsonized bacteria could be depressed [36]. In this study, CL inhibition of the OZ-stimulated neutrophils isolated from hypercapnic rabbits supports these observations. Extracellular followed by a rapid intracellular $\mathrm{pH}$ decrease may also directly activate neutrophils, although at a level $(\mathrm{pH}=6.5)$ far below those observed in this study [37]. Eventually, in this study, no impact of hypercapnia on systemic oxidative stress, an important biomarker of experimental endotoxemia, could be observed [38].

With regard to experimental conditions applied in this study, mild stretch respiratory pattern could promote a systemic inflammatory response; however, in a rabbit model of pneumonia no relevant systemic reaction could be observed until $8 \mathrm{~h}$ of mechanical ventilation [39]. Similar end-point blood pressure values observed in both groups in line with previous findings [18] despite systemic $\mathrm{pH}$ differences suggest that acidosis was of minor significance for circulatory events in this experimental model. Bradycardia, also comparable between the groups, could indicate parasympathetic shift which may contribute to the anti-inflammatory compensatory response, of possible importance also for oxidative phenomena [40].

\section{Limitations}

We are aware of limitations. First, we addressed experimental conditions with moderate elevation of $\mathrm{pCO}_{2}$ for a short time; in postresuscitation patients values of $\mathrm{PaCO}_{2}$ between 60 and $70 \mathrm{mmHg}$, as applied in this study, turned out to be optimal concerning the neurological outcome. Second, we also applied a moderate LPS dose, which does not reflect severe inflammatory conditions; data suggest that severity of the overall inflammatory response may portend a negative impact of hypercapnia on the final outcome $[5,8]$. Third, the TBARS assay as a biomarker of lipid peroxidation and oxidative stress despite its broad application has relatively low specificity and should be interpreted with caution. Fourth, neutrophils constitute a highly heterogenous population and our results reflect an overall response of these cells. Further research should discriminate between neutrophil phenotypes under endotoxin challenge and assess the influence of hypercapnia on ROS generation by particular cell subsets.

\section{Conclusions}

Taken together, acute isooxic moderate hypercapnia in endotoxemic rabbits influences neither peripheral leukocyte and neutrophil count patterns nor neutrophil ROS generation. Our results support safety of short-term hypercapnia of moderate degree in inflammatory conditions regarding its impact on innate immunity. A putative regulatory role of hypercapnia considering further inflammatory implications under conditions of endotoxemia warrants further studies.

\section{Acknowledgments}

The study was supported by resources of the Poznan University of Medical Sciences.

The authors declare no conflict of interest.

\section{References}

1. Curley G, Hayes M, Laffey JG (2011): Can 'permissive' hypercapnia modulate the severity of sepsis-induced ALI/ ARDS? Crit Care 15: 212.

2. Clyde TP, Coletta M, Jones C, et al. (2018): Effects of hypercapnia in sepsis: protocol for a systematic review of clinical and preclinical data. Syst Rev 7: 171.

3. Shigemura M, Lecuona E, Sznajder JI, et al. (2017): Effects of hypercapnia on the lung. J Physiol 595: 2431-2437.

4. Fuller BM, Mohr NM, Drewry AM, et al. (2017): Partial pressure of arterial carbon dioxide and survival to hospital discharge among patients requiring acute mechanical ventilation: A cohort study. J Crit Care 41: 29-35.

5. Tiruvoipati R, Pilcher D, Buscher H, et al. (2017): Effects of hypercapnia and hypercapnic acidosis on hospital mortality in mechanically ventilated patients. Crit Care Med 45: e649-e656.

6. Roberts BW, Mohr NM, Ablordeppey E, et al. (2018): Association between partial pressure of arterial carbon dioxide and survival to hospital discharge among patients diagnosed with sepsis in the emergency department. Crit Care Med 46: e213-e220.

7. Fuchs H, Rossmann N, Schmid MB, et al. (2017): Permissive hypercapnia for severe acute respiratory distress syndrome in immunocompromised children: A single center experience. PLoS One 12: e0179974.

8. Nin N, Muriel A, Peńuelas O, et al. (2017): Severe hypercapnia and outcome of mechanically ventilated patients with moderate or severe acute respiratory distress syndrome. Intensive Care Med 43: 200-208.

9. Leliefeld PH, Wessels CM, Leenen LP, et al. (2016): The role of neutrophils in immune dysfunction during severe inflammation. Crit Care 20: 73.

10. Gates KL, Howell HA, Nair A, et al. (2013): Hypercapnia impairs lung neutrophil function and increases mortality in murine pseudomonas pneumonia. Am J Respir Cell Mol Biol 49: 821-828.

11. Ni Chonghaile M, Higgins BD, Costello JF, Laffey JG (2008): Hypercapnic acidosis attenuates severe acute bacterial pneumonia-induced lung injury by a neutrophil-independent mechanism. Crit Care Med 36: 3135-3144.

12. Shimotakahara A, Kuebler JF, Vieten G, et al. (2008): Carbon dioxide directly suppresses spontaneous migration, chemotaxis, and free radical production of human neutrophils. Surg Endosc 22: 1813-1817.

13. Dean JB (2010): Hypercapnia causes cellular oxidation and nitrosation in addition to acidosis: implications for $\mathrm{CO} 2$ chemoreceptor function and dysfunction. J Appl Physiol 108: 1786-1795.

14. Billert H, Drobnik L, Podstawska D, et al. (2003): Oxidative metabolism of peripheral blood neutrophils in experimental acute hypercapnia in the mechanically ventilated rabbit. Vascul Pharmacol 40: 119-125. 
15. Beachy JC, Weisman LE (1993): Acute asphyxia affects neutrophil number and function in the rat. Crit Care Med 21: 1929-1934.

16. Vernon PJ, Schaub LJ, Dallelucca JJ, et al. (2015): Rapid detection of neutrophil oxidative burst capacity is predictive of whole blood cytokine responses. PLoS One 10: e0146105.

17. Vaure C, Liu Y (2014): A comparative review of toll-like receptor 4 expression and functionality in different animal species. Front Immunol 5: 316.

18. Kuebler WM, Borges J, Sckell A, et al. (2000): Role of L-selectin in leukocyte sequestration in lung capillaries in a rabbit model of endotoxemia. Am J Respir Crit Care Med 161: 36-43.

19. Kregenow DA, Rubenfeld GD, Hudson LD, Swenson ER (2006): Hypercapnic acidosis and mortality in acute lung injury. Crit Care Med 34: 1-7.

20. Lang JD, Figueroa M, Sanders KD, et al. (2005): Hypercapnia via reduced rate and tidal volume contributes to lipopolysaccharide-induced lung injury. Am J Respir Crit Care Med 171: 147-57.

21. Issekutz AC, Issekutz TB (1988): Cellular and vascular phenomena in inflammation. Meth Enzymol 162: 301-20.

22. Allen RC. (1986): Phagocytic leukocyte oxygenation activities and chemiluminescence: a kinetic approach to analysis. Meth Enzymol 133: 449-493.

23. Labedzka M, Gulyas H, Schmidt N, Gercken G (1989): Toxicity of metallic ions and oxides to rabbit alveolar macrophages. Environment Res 48: 255-74.

24. Macfarlane DE (1981): The effects of methyl mercury on platelets: induction of aggregation and release via activation of the prostaglandin synthesis pathway. Mol Pharmacol 19: 470-476.

25. van-Eeden SF, Kitagawa Y, Klut ME, et al. (1997): Polymorphonuclear leukocytes released from the bone marrow preferentially sequester in lung microvessels. Microcirculation 4: 369-80

26. Alarcón P, Conejeros I, Carretta MD, et al. (2011): D-lactic acid interferes with the effects of platelet activating factor on bovine neutrophils. Vet Immunol Immunopathol 144: 68-78.

27. Etheredge EE, Spitzer JA (1993): Chronic endotoxemia reversibly alters respiratory burst activity of circulating neutrophils. J Surg Res 55: 261-268.

28. Pillay J, Ramakers BP, Kamp VM, et al. (2010): Functional heterogeneity and differential priming of circulating neutrophils in human experimental endotoxemia. J Leukoc Biol 88: 211-20.

29. van Leeuwen HJ, Van Der Tol M, Van Strijp JA, et al. (2005): The role of tumour necrosis factor in the kinetics of lipopolysaccharide-mediated neutrophil priming in whole blood. Clin Exp Immunol 140: 65-72.

30. Nguyen GT, Green ER, Mecsas J (2017): Neutrophils to the ROScue: mechanisms of NADPH oxidase activation and bacterial resistance. Front Cell Infect Microbiol 7: 373.

31. Frazier LW (1991): Stimulation of membrane phospholipid metabolism by agents that increase acidification in toad urinary bladder. Proc Soc Exp Biol Med 197: 208-213.

32. Yokoyama M, Ishida H, Okita T, et al. (2003): Oncological effects of insufflation with different gases and a gasless procedure in rats. Surg Endosc 17: 1151-1155.

33. Pallister I, Topley N (2004): Chemiluminescence: comparison of whole blood with isolated polymorphonuclear leukocytes after major trauma. J Trauma 57: 347-351.

34. Volk J, Kleine HD, Buthmann U, Freund M (2000): Oxidative burst measurement in patients treated with cytostatics: influ- ence of G-CSF and role as a prognostic factor. Ann Hematol 79: 187-197.

35. Wittmann S, Rothe G, Schmitz G, Fröhlich D (2004): Cytokine upregulation of surface antigens correlates to the priming of the neutrophil oxidative burst response. Cytometry A 57: 53-62.

36. Lardner A (2001): The effects of extracellular $\mathrm{pH}$ on immune function. J Leukoc Biol 69: 522-530.

37. Martinez D, Vermeulen M, Trevani A, et al. (2006): Extracellular acidosis induces neutrophil activation by a mechanism dependent on activation of phosphatidylinositol 3-kinase/Akt and ERK pathways. J Immunol 176: 1163-1171.

38. Steven S, Dib M, Roohani S, et al. (2017): Time response of oxidative/nitrosative stress and inflammation in LPS-induced endotoxaemia-a comparative study of mice and rats. Int J Mol Sci 18: E2176.

39. Barbar SD, Pauchard LA, Bruyčre R, et al. (2006): Mechanical ventilation alters the development of staphylococcus aureus pneumonia in rabbit. PLoS One 11: e0158799.

40. Li DJ, Fu H, Tong J, et al. (2018): Cholinergic anti-inflammatory pathway inhibits neointimal hyperplasia by suppressing inflammation and oxidative stress. Redox Biol 15: 22-33. 